\title{
Mineral Economics/Raw Materials Report 30th anniversary
}

\author{
Magnus Ericsson $^{1}$
}

Published online: 28 February 2017

(C) Springer-Verlag Berlin Heidelberg 2017

In 2017, Mineral Economics celebrates its 30th anniversary. It has been a long and winding journey noted not least in the fact that if we had progressed according to the calendar, we should have reached volume 37! The name of the journal has also changed: Raw Materials Report; Journal of Mineral Policy, Business and Environment; Minerals \& Energy and lastly Mineral Economics. But metals and mining and their fundamental role in global social and economic development have always remained in focus.

In 1980, a non-hierarchical "Editorial Group" consisting of nine persons optimistically started the journal. All of us were active in the Raw Materials Group (RMG) in Stockholm, Sweden. We had studied the international mining industry and its economic, social, and political impacts from the beginning of the 1970s. Our starting point was a global economic and political analysis. We also tried to understand the situation in Sweden, where the iron ore industry was in crisis. Gradually, we got convinced that an international network was necessary to better understand the dynamics of mining in Sweden and the role of metals worldwide. It was easy to start the journal, initially called Raw Materials Report, but we had not grasped the difficulties we would meet. Without internet, and only after some years having resources enough to get a fax machine, communications around the world were slow and expensive. In those days, we not only collected material and wrote articles but also typeset and did the graphic design with scalpels and glue. We photographed and retouched each spread before sending an issue to the printer. When the printed copies were delivered, we put address labels on envelopes and finally drove to the post office to send the copies off by air mail to all subscribers. Afterwards, we did the

Magnus Ericsson

magnus@gladtjarnen.se

1 Luleå University of Technology, Luleå, Sweden

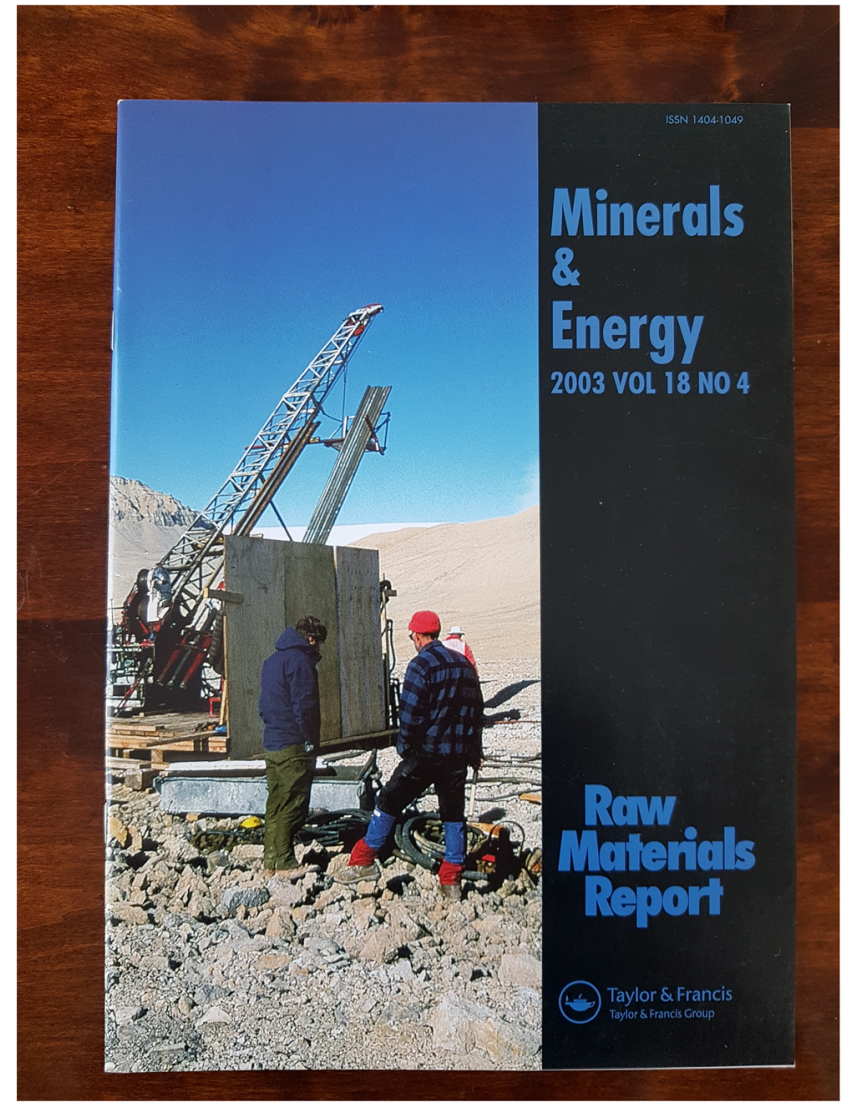

2000-2008

admin work and most importantly marketing, trying to reach new readers and get additional subscribers. Support from the Swedish government (Swedish State Council for Culture) helped initially but without all the un-paid work and dedication of all our contributors and friends, the journal would have died after the first issues. This network of friends and colleagues is still alive and remains our firm foundation for future developments.

In the beginning, we also had cultural ambitions. In the first issue, we translated a chapter from the novel "The men who 


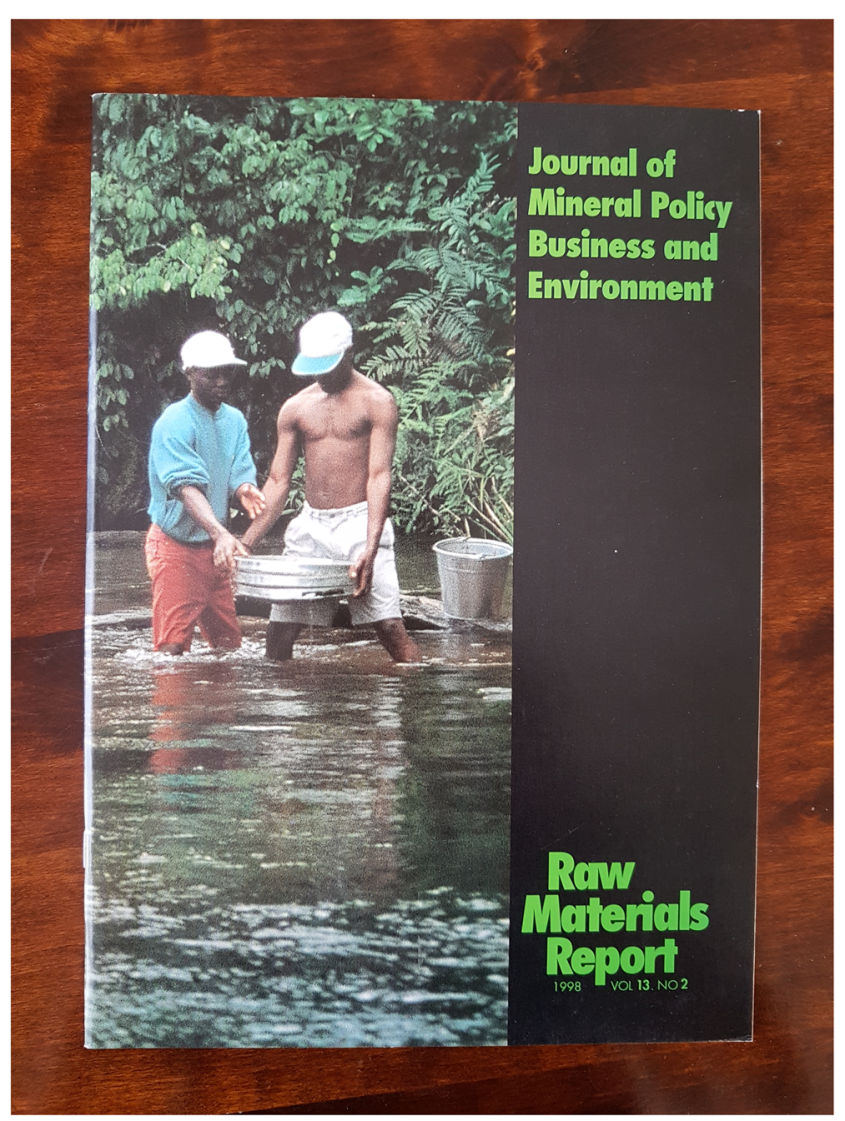

1995-1999

did it," which was written by the Swedish author Ernst Didring in 1914. It was the first volume in a trilogy about the painful birth of the iron ore mine in Kiruna. Over the years, we have had to give up these aspirations and become a traditional scientific journal.

But we are still alive and kicking. Since 1998, we cooperate closely with Luleå University of Technology and their support is vital for us and provides an excellent basis to grow from. In 2017, we hope to broaden our international cooperation further and revitalize our links globally and with Europe. We have invited universities outside the Nordic region to become partners in publishing Mineral Economics. We will also extend the Editorial Advisory Board, and I am happy to announce that Dr. Joanna Kulczycka from AGH University of Science and Technology, Cracow, Poland and Masuda Nobuyuki at Akita University, Japan, have already agreed to join.

With this special issue, we begin our reporting from the many EU research projects, which have been started during the last years. Please see the Foreword and Introduction in the

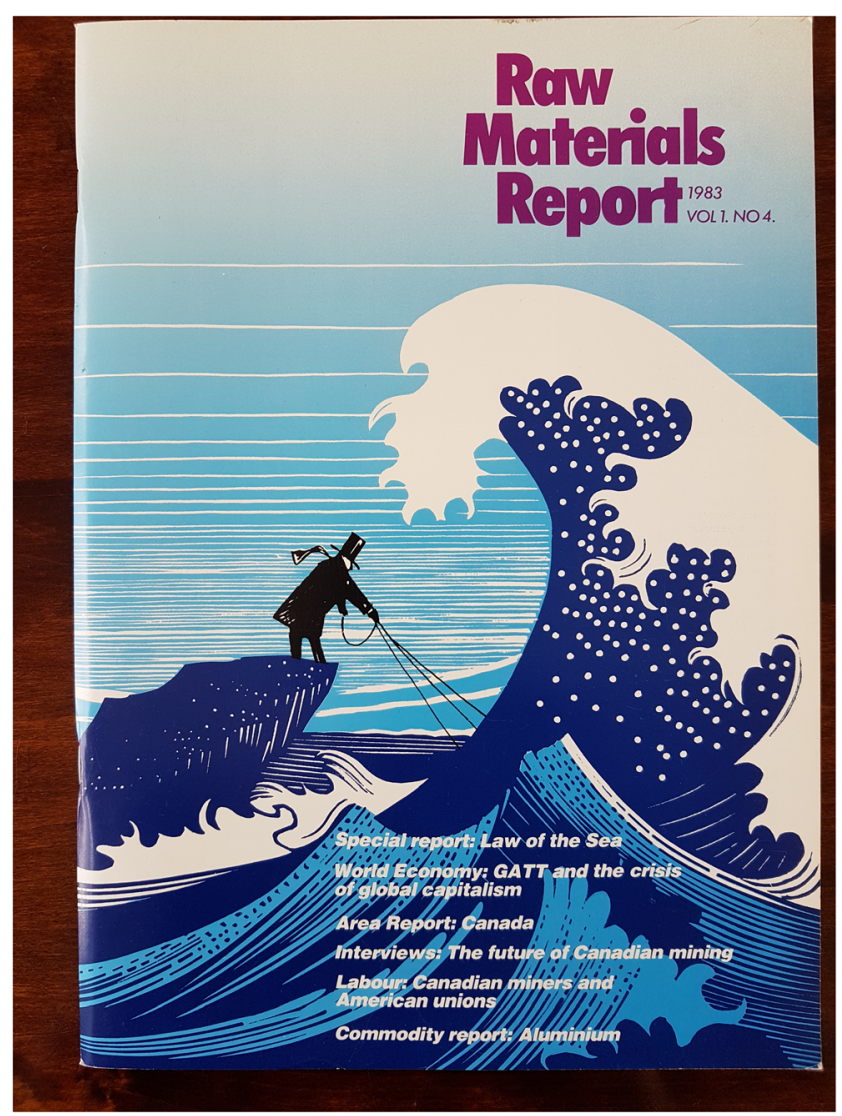

1980-1994

following pages. These projects span over a wide area of both economics, policy, law, and technology. We expect a host of interesting reports and an intensive discussion to grow out of all these projects.

In one of our forthcoming issues, we intend to focus on exploration and I would like to invite papers on all aspects of this broad topic. One of our advantages as a relatively small journal is our fast turn-around and quick publishing after completed reviews. We welcome your contribution whether a full length paper, a brief report, or a comment on something already published, which you do or do not agree with.

After long and dedicated service, one of our two Managing Editors is stepping down. I want to thank Thomas Ejdemo for his efforts over the years. He has however promised to continue to support the journal but in a less demanding position.

Magnus Ericsson,

Editor-in-Chief

One of the founders in the Editorial Group of 1980. 